\title{
A nonsense mutation in PLD4 is associated with a zinc deficiency-like syndrome in Fleckvieh cattle
}

Simone Jung ${ }^{1 \dagger}$, Hubert Pausch ${ }^{1 \dagger}$, Martin C Langenmayer ${ }^{3}$, Hermann Schwarzenbacher ${ }^{4}$, Monir Majzoub-Altweck ${ }^{3}$, Nicole S Gollnick ${ }^{2}$ and Ruedi Fries ${ }^{1 *}$

\begin{abstract}
Background: Bovine hereditary zinc deficiency (BHZD) is an autosomal recessive disorder of cattle, first described in Holstein-Friesian animals. Affected calves suffer from severe skin lesions and show a poor general health status. Recently, eight calves with the phenotypic appearance of BHZD have been reported in the Fleckvieh cattle population.

Results: In spite of the similar disease phenotypes, SLC39A4, the gene responsible for BHZD in Holstein-Friesian was excluded as underlying gene for the disorder in the affected Fleckvieh calves. In order to identify the disease-associated region, genotypes of eight affected calves obtained with the Illumina BovineHD BeadChip comprising 777,962 SNPs were contrasted with the genotypes of 1,339 unaffected animals. A strong association signal was observed on chromosome $21\left(P=5.87 \times 10^{-89}\right)$. Autozygosity mapping in the eight affected animals revealed a common segment of extended homozygosity encompassing 1,023 kb (BTA 21: 70,550,045 - 71,573,501). This region contains 17 genes/transcripts, among them two genes encoding gastro-intestinal zinc transporters (CRIP1, CRIP2). However, no mutation that was compatible with recessive inheritance could be detected in these candidate genes. One of the affected calves was re-sequenced together with 42 unaffected Fleckvieh animals. Analysis of the sequencing data revealed a nonsense mutation (p.W215X) in a phospholipase encoding gene (PLD4) as candidate causal polymorphism. To confirm the causality, genotypes of the p.W215X-mutation were obtained from 3,650 animals representing three different breeds. None of the unaffected animals was homozygous for the defect allele, while all eight affected calves were homozygous. The deleterious effect of the mutation is manifested in a significantly lower survival rate of descendants from risk matings when compared with the survival rate of descendants from non-risk matings. The deleterious allele has an estimated frequency of $1.1 \%$ in the Fleckvieh population.

Conclusion: Our results provide strong evidence that a newly identified recessive disorder in the Fleckvieh population is caused by a nonsense mutation in PLD4, most likely resulting in an impaired function of the encoded protein. Although the phenotype of affected calves strongly resembles BHZD, a zinc deficiency resulting from malabsorption is unlikely to be responsible for the diseased Fleckvieh calves.
\end{abstract}

Keywords: Autozygosity mapping, Next-generation sequencing, Recessive inheritance, Zinc deficiency-like, Fleckvieh, Nonsense mutation, PLD4

\footnotetext{
* Correspondence: ruedi.fries@tum.de

'Equal contributors

${ }^{1}$ Chair of Animal Breeding, Technische Universitaet Muenchen, 85354

Freising, Germany

Full list of author information is available at the end of the article
}

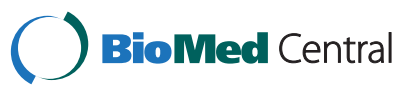

(c) 2014 Jung et al.; licensee BioMed Central Ltd. This is an Open Access article distributed under the terms of the Creative Commons Attribution License (http://creativecommons.org/licenses/by/4.0), which permits unrestricted use, distribution, and reproduction in any medium, provided the original work is properly credited. The Creative Commons Public Domain Dedication waiver (http://creativecommons.org/publicdomain/zero/1.0/) applies to the data made available in this article, unless otherwise stated. 


\section{Background}

So far, 415 congenital defects have been identified in cattle, 109 of them being inherited in an autosomal recessive mode [1]. The widespread use of elite sires by means of artificial insemination makes cattle populations highly susceptible to the propagation of recessively inherited disorders [2]. However, the availability of dense SNP genotyping arrays facilitates the rapid identification of underlying genomic regions [3].

This paper details a disorder that resembles Bovine Hereditary Zinc Deficiency (BHZD, OMIA 000593-9913), an autosomal recessive condition, primarily seen in Holstein-Friesian calves [4]. This disease is characterized by an impaired function of the immune system, growth retardation and skin alterations as a result of a deficient gastrointestinal zinc absorption [5]. While affected animals are born without apparent clinical symptoms, first skin lesions emerge between the age of four and eight weeks [4]. Impairment of immune functions makes affected animals more susceptible to infectious pathogens and leads to an increased incidence of common calf diseases, e.g., enteritis and pneumonia [6]. Highlydosed oral zinc supplementation ameliorates clinical symptoms in affected Holstein-Friesian animals, however, if untreated, BHZD is lethal [5]. Inherited zinc absorption disorders, caused by mutations in the zinc transporter encoding gene SLC39A4, have been reported for several mammalian species, including mouse and human [7-9]. In Holstein-Friesian cattle, BHZD is caused by a splice-site variant in $S L C 39 A 4$ [8]. Hereditary zinc absorption disorders have not yet been described in the Fleckvieh population.

Here we show that a phenotype of Fleckvieh calves that resembles BHZD is not caused by a defective zinc transporter gene. Genome-wide association analysis, autozygosity mapping and analysis of whole-genome sequencing data enabled to identify a nonsense mutation in the phospholipase $\mathrm{D}$ family member 4 encoding gene (PLD4) which is likely causal for the observed phenotype.

\section{Results}

\section{Phenotypic manifestation of the defect}

Seven calves between the age of seven and 17 weeks with severe skin lesions and poor general health status were admitted to the Clinic of Ruminants. Clinical findings such as scaling and crusting of skin and secondary adhesion of hair were most evident around the muzzle, the eyes, above the sternum and on the extremities (Figure 1A,B). Skin of the inguinal region was seborrhoeic and all palpable lymph nodes were enlarged. The calves suffered from erosions in the interdigital spaces and erosive or ulcerative lesions of the oral mucosa (Figure 1C). All animals were underdeveloped in height and weight and had a history of recurring diarrhoea and pneumonia. Two of the

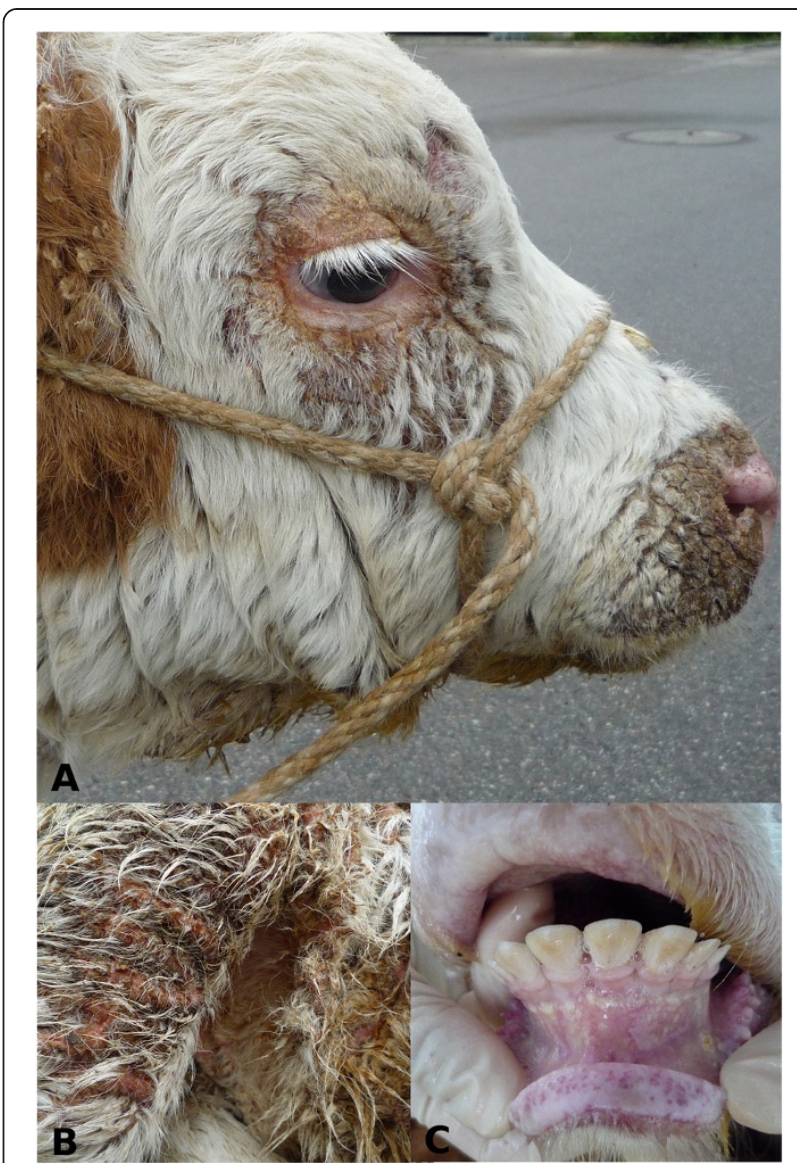

Figure 1 Phenotypic manifestation of the newly identified disease in Fleckvieh calves. A two month old male Fleckvieh calf with distinct scaling and crusting of the skin around the muzzle, eyes (A) and in the femoral region of the right hind leg (B). Alterations due to seborrheic dermatitis can be noted in the inguinal region (B). Erosive lesions of the oral mucosa in a 4.4-month old female patient (C).

calves were supplemented with dietary zinc (750 mg/day), but did not respond to the treatment. Due to the advanced state of the disease and with no prospect for improvement, all animals were euthanized and subjected to necropsy.

In hematoxylin and eosin $(\mathrm{H} \& \mathrm{E})$ stained sections, the skin of the affected animals displayed a mild to severe chronic dermatitis with serocellular crusting and partial superficial bacterial colonization. Multiple intracorneal and intraepidermal accumulations of serum as well as multiple ulcerations and epidermal necroses were present. The epidermis was severely oedematous in multiple sections (Figure 2). Dermis and epidermis were diffusely infiltrated by a moderate to high number of neutrophils. Besides moderate oedema, the dermis exhibited infiltration with lymphocytes and plasma cells to a limited extent. In the cases where the thymus was examined, decreased thymic cellularity and poorly demarcated cortex-medullaborder were observed. Further, the clinical diagnoses of 


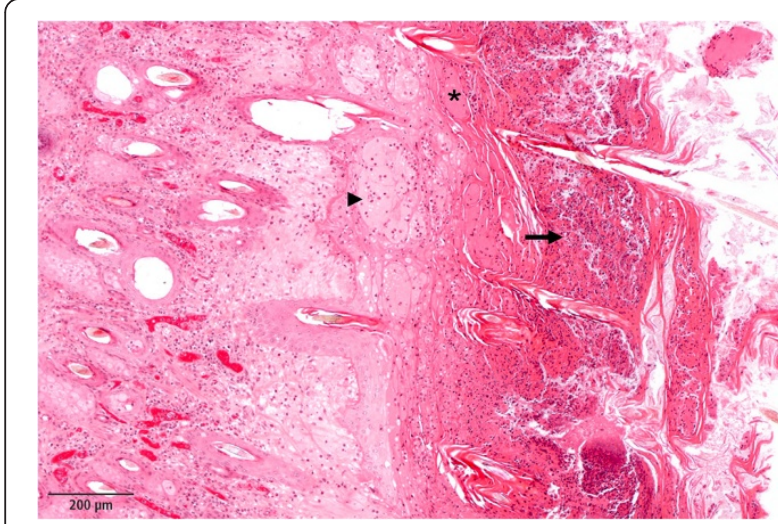

Figure 2 Hematoxylin and eosin (H\&E) stained dermal section of an affected calf. Severe epidermal vesiculation (arrowhead), multiple intracorneal serum accumulations (asterisk), serocellular crusts (arrow) and diffuse dermal oedema and inflammation, plastic embedding technique, H\&E stain.

enteritis and pneumonia were confirmed during the pathological examination.

In addition to the seven cases described above, another Fleckvieh calf (eleven weeks old) with severe alterations of the skin and diarrhoea was reported by a veterinarian. The described lesions were very similar to the phenotype of the previously reported seven calves. The calf received dietary zinc $(750 \mathrm{mg} /$ day) for a period of two weeks. However, the overall condition of the animal did not improve and the calf died at the age of 13 weeks.

Inspection of the pedigrees of the affected calves revealed a common ancestor suggesting a genetic background. On the basis of the patients' history, the clinical and pathological findings, the calves were tentatively diagnosed to suffer from BHZD.

\section{Analysis of SLC39A4 - the gene causing BHZD in Holstein-Friesian}

Mutations in SLC39A4 are known to cause defects resembling the phenotypic appearance of the eight affected Fleckvieh calves in various species including cattle [8]. SLC39A4 is located at the proximal region of bovine chromosome 14 (BTA 14: 1,719,732 bp $1,724,221 \mathrm{bp})$. The gene was re-sequenced in a casecontrol panel consisting of all affected animals, all available dams and sires and randomly selected, unaffected control animals. Totally $\sim 7 \mathrm{~kb}$ of genomic sequence was screened, resulting in the detection of ten SNPs (Additional file 1). The mutation causing BHZD in Holstein-Friesian was not present in the diseased animals and none of the detected polymorphisms was associated with the disease phenotype, nor was any of the polymorphic sites compatible with the supposed pattern of recessive inheritance.

\section{Identification of the disease-associated region}

Since the analysis of SLC39A4 did not reveal a potentially causal mutation, we applied an array-based approach to identify the underlying genomic region. The eight affected calves together with 1,339 unaffected Fleckvieh bulls were genotyped with the Illumina BovineHD BeadChip. A genome-wide association study using genotypes of 644,450 SNPs revealed a strong association signal on BTA 21. Eighty-two SNPs located within an $18.19 \mathrm{Mb}$ interval from $53,140,245 \mathrm{bp}$ to $71,333,740 \mathrm{bp}$ were significantly associated $\left(\mathrm{P}<7.88 \times 10^{-8}\right)$ (Figure $\left.3 \mathrm{~A}\right)$. The most prominent association signal $\left(\mathrm{P}=5.87 \times 10^{-89}\right)$ was observed for BovineHD4100015383, located at $69,873,257$ bp. As expected, the proximal region of BTA 14 harbouring SLC39A4 did not show association at all.

Autozygosity mapping within the distal region of BTA 21 revealed a common $1,023 \mathrm{~kb}$ segment of extended homozygosity in the eight affected animals (70,550,045 bp - 71,573,501 bp) (Figure 3B). However, one out of 1,339 animals of the control group was homozygous for the disease-associated region as well. Signal intensities obtained from high-density genotyping revealed no indication for the presence of large structural variants (deletions, copy number variations) within the associated region (Additional file 2).

The frequency of the associated haplotype was estimated in a sample of 10,355 unaffected Fleckvieh animals. Among them, 380 were heterozygous carriers and three were homozygous, yielding a frequency for the associated haplotype of $1.86 \%$. The haplotype distribution shows no deviation from the Hardy-Weinberg equilibrium $(\mathrm{P}=0.75)$.

\section{Analysis of CRIP1 and CRIP2 - genes with similar function as SLC39A4}

The segment of extended homozygosity contains 17 transcripts and protein encoding genes, respectively (UMD3.1 annotation [10], Figure 3C). Two of these genes encode cysteine-rich proteins (CRIP1 and CRIP2). CRIPs are highly expressed in the intestine [11] and are essential for zinc absorption as well as for immune system related cytokine regulation $[12,13]$. Thus, they represent excellent candidate genes for the observed disease phenotype.

CRIP1 and CRIP2 (71,390,596 bp - 71,392,110 bp and $71,365,858$ bp $-71,382,666 \mathrm{bp}$, respectively) were resequenced in the case-control panel. We screened $\sim 6 \mathrm{~kb}$ of the genomic sequence of CRIP1, resulting in the detection of 18 SNPs and two insertion/deletion (InDel) polymorphisms (Additional file 1). Approximately $8 \mathrm{~kb}$ of the genomic sequence of CRIP2 were screened, resulting in the identification of 30 SNPs (Additional file 1). None of the variants in CRIP1 and CRIP2 was compatible with the 


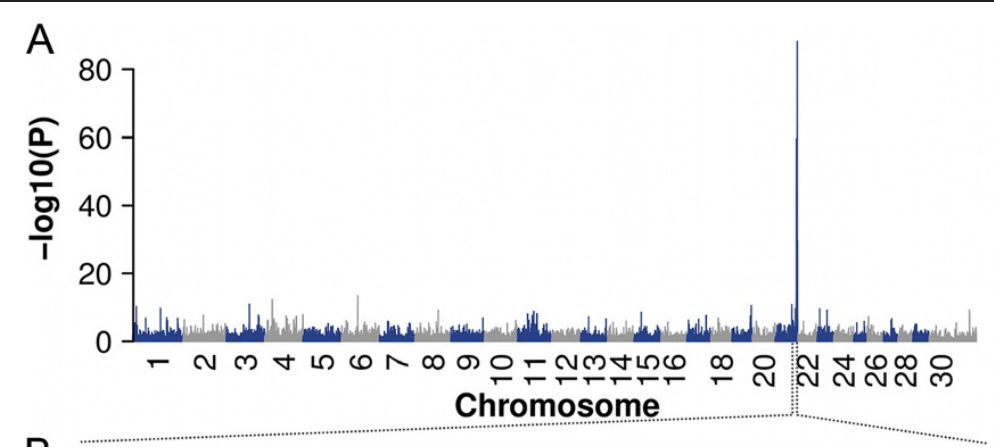

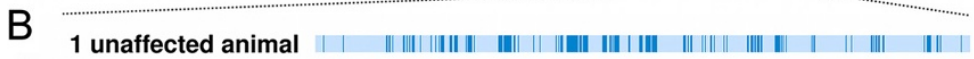
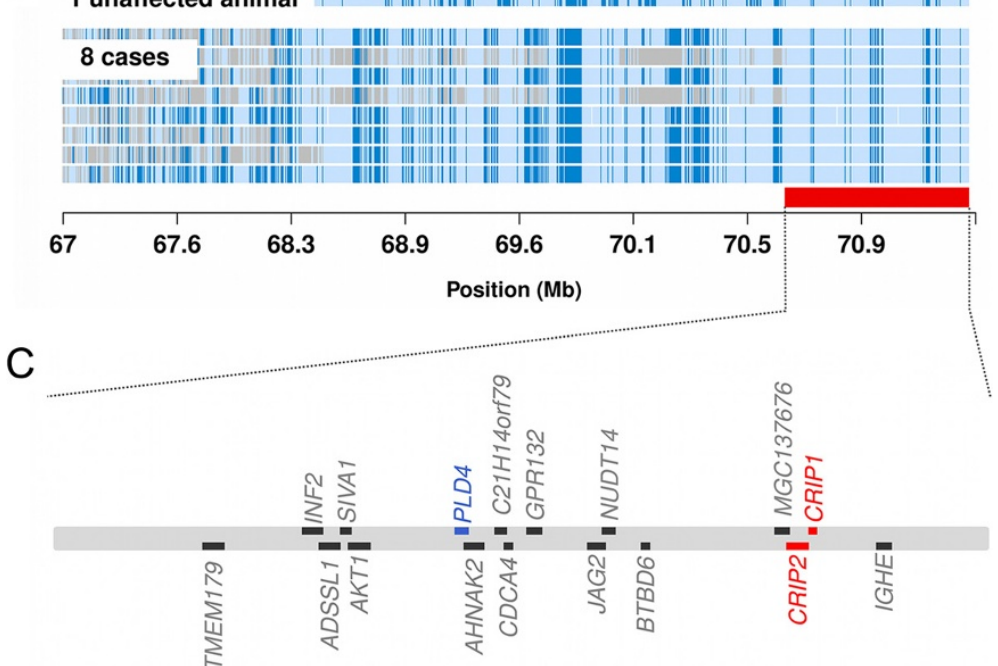

Figure 3 Mapping of the locus for a zinc deficiency-like syndrome in the Fleckvieh population. Association of 644,450 SNPS with the affection status of eight affected and 1,339 unaffected Fleckvieh animals (A). P values were obtained by fitting a linear mixed model. Autozygosity mapping in eight affected calves and one unaffected animal (B). Blue and pale blue represent homozygous genotypes (AA and BB), heterozygous genotypes (AB) are displayed in light grey. The red bar indicates the common segment of homozygosity. Note that one unaffected animal is also homozygous for the segment of extended homozygosity. The segment of extended homozygosity encompasses 17 transcripts/genes among them CRIP1, CRIP2 and PLD4 (C).

disease phenotype. Thus, variation in the two positional and functional candidate genes is likely not causal for the observed disease.

\section{Identification of the underlying mutation by exploiting whole-genome sequencing data}

In a next attempt to identify the causal mutation, one of the affected calves (id =58953) and one of the unaffected homozygous animals (id $=58952$ ) were re-sequenced together with 41 animals of the FV population [14]. Multi-sample variant calling yielded genotypes for 7,660 polymorphic sites within the $1,032 \mathrm{~kb}$ disease-associated segment at the distal end of BTA 21. Sequence coverage in the disease-associated region averaged $5.19 \mathrm{x}$ and $3.95 x$ for 58953 and 58952, respectively with $87.95 \%$ and $80.60 \%$ of the positions being covered with more than one read. Haplotype analysis revealed that none of 41 re-sequenced control animals carried the diseaseassociated haplotype. Three SNPs and one InDel were compatible with recessive inheritance, i.e., they were homozygous for the alternative allele (aa) in the affected calf, heterozygous (Aa) or homozygous for the reference allele (AA) in 58952 and homozygous for the reference allele (AA) in all 41 remaining animals (Table 1). None of the four polymorphisms were located within the coding regions of two candidate genes CRIP1 and CRIP2.

The compatible InDel-polymorphism (rs381259516) is also segregating among 191 non-Fleckvieh animals (128 Holstein, 15 Jersey, 48 Angus), which have been sequenced in the context of the 1000 bull genomes project [15]. No regulatory or functional consequence was predicted for the compatible SNP (rs385301007) in intron 30 of LOC100299595. Manual re-annotation of INF2 revealed that the presumed missense mutation ( $r$ 384306864) is not located within the coding region of the gene (Additional file 3). Therefore, these three variants were excluded as being causal for the described 
Table 1 Genotypes of four variants compatible with recessive inheritance

\begin{tabular}{|c|c|c|c|c|c|c|c|c|}
\hline \multirow[t]{2}{*}{ Chr } & \multirow[t]{2}{*}{ Position } & \multirow[t]{2}{*}{ NCBI reference ID } & \multirow[t]{2}{*}{ Variant } & \multicolumn{3}{|c|}{ Genotypes } & \multirow[t]{2}{*}{ Gene } & \multirow[t]{2}{*}{ Effect } \\
\hline & & & & Calf & 58952 & 41 animals & & \\
\hline 21 & $70,679,787$ & rs381259516 & InDel & del/del & $\mathrm{del} / \mathrm{G}$ & $\mathrm{G} / \mathrm{G}$ & intergenic & - \\
\hline 21 & $70,842,696$ & rs384306864 & SNP & $\pi$ & CC & CC & INF2 & p.H1231Y \\
\hline 21 & $71,001,232$ & rs378824791 & SNP & AA & GG & GG & PLD4 & p.W215X \\
\hline 21 & $71,315,111$ & rs385301007 & SNP & $\pi$ & CC & CC & LOC100299595 (=PACS2) & intron 30 \\
\hline
\end{tabular}

Whole-genome re-sequencing of 43 animals revealed 7,660 polymorphic sites within the 1,032 kb segment on BTA 21 . Among them, three SNPs and one InDel agree with the supposed inheritance pattern (i.e., the affected calf is homozygous for the alternative allele and the controls are heterozygous or homozygous for the reference allele). 58952 is a healthy animal being homozygous for the disease-associated haplotype.

phenotype. Only a point mutation in exon 6 of PLD4, resulting in a premature stop codon (c.G645A, p.W215X, BTA 21:71,001,232 bp, rs378824791) (Figure 4, Additional file 3), was retained as candidate causal mutation. The resulting protein is shortened by 273 amino acids and lacks essential domains for enzymatic activity [16,17]. The mutation was confirmed by Sanger sequencing (Additional file 4).

\section{Validation of the p.W215X-mutation in PLD4}

Genotypes of the p.W215X-mutation (rs378824791) were obtained for 3,650 animals representing three different breeds (Fleckvieh: 3,088, Braunvieh: 280, Holstein-Friesian: 282). All eight affected calves were homozygous for the mutation, while none of the unaffected animals was homozygous (Table 2). The mutation does not segregate in Braunvieh and Holstein-Friesian. Three unaffected animals identified as homozygous for the disease-associated haplotype via array-derived genotypes were heterozygous and homozygous for the reference allele, respectively. We obtained genotypes for 169 animals that are heterozygous carriers of the disease-associated haplotype (identified via array-derived genotypes). Among them, only 100 carry the mutation while 69 are homozygous for the reference allele. The frequency of the associated haplotype was estimated to be $1.86 \%$ based on array-derived genotypes (see above). However, only $59.5 \%$ of the animals carrying the disease haplotype also carry the p.W215X-mutation. Thus, the frequency of the defect allele amounts to $1.1 \%$ in the Fleckvieh population.

\section{Survival rate of progeny descending from bulls carrying the p.W215X-mutation}

In order to study the effect of the deleterious allele on the population level, we estimated the survival rate of the descendants from different mating types with regard to the p.W215X-genotype in sires and maternal grandsires. If both, sire and maternal grandsire are heterozygous for the p.W215X-mutation, the probability of the resulting calf being homozygous is $12.5 \%$. The survival rate of calves descending from such matings $(\mathrm{N}=1,213)$ is significantly lower $\left(\mathrm{P}=2.971 \times 10^{-8}\right)$ compared to the survival rate of calves descending from non-risk matings across all age classes, as expected in the case of recessive inheritance of the deleterious allele (Figure 5). At day $300,16.63 \%$ of the calves resulting from risk matings have perished, while the mortality of calves descending from non-risk matings is $9.66 \%$ only (Table 3 ).

\section{Discussion}

Eight calves with severe skin lesions and poor general health status were recently identified in the Fleckvieh cattle population. Clinical and pathological examinations of affected animals showed striking similarities to findings described for individuals suffering from zinc deficiency in cattle $[4,6,18]$ and humans [19]. Inherited zinc absorption disorders, resulting from mutations in SLC39A4 [8], can be ameliorated by highly-dosed oral zinc supplementation [5]. However the Fleckvieh calves did not respond to oral zinc supplementation. Variants in SLC39A4 could be excluded from being causal for the phenotype of the affected Fleckvieh animals, suggesting

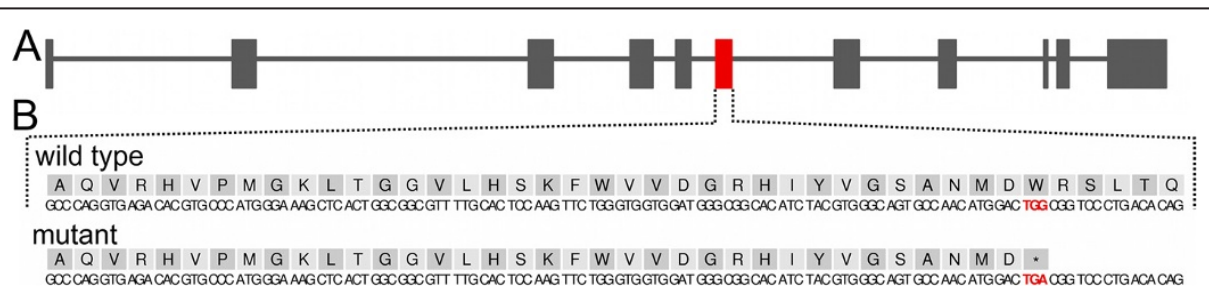

Figure 4 A nonsense mutation in PLD4 is perfectly associated with the disease phenotype. Genomic structure of bovine PLD4 (A). Grey boxes represent exons. The red box represents exon 6 including rs378824791, introducing a premature stop codon. Genomic and protein sequence of exon 6 of PLD4 (B). The affected codon (p.W215X, TGG $\rightarrow$ TGA) is highlighted with red colour. 
Table 2 Genotypes for the p.W215X-mutation in PLD4 for 3,650 animals

\begin{tabular}{|c|c|c|c|c|c|}
\hline \multirow[t]{2}{*}{ Breed } & \multirow[t]{2}{*}{ Animal group } & \multirow[t]{2}{*}{ Samples } & \multicolumn{3}{|c|}{ Genotypes for p.W215X } \\
\hline & & & AA & AG & GG \\
\hline \multirow[t]{6}{*}{ FV } & Affected calves & 8 & 8 & - & - \\
\hline & Dams of the calves & 6 & - & 6 & - \\
\hline & Unaffected animals being homozygous for the disease-associated haplotype & 3 & - & 2 & 1 \\
\hline & Animals carrying the disease-associated haplotype & 169 & - & 100 & 69 \\
\hline & Animals not carrying the disease-associated haplotype & 1,777 & - & - & 1,777 \\
\hline & Animals with unknown haplotype status & 1,125 & - & 51 & 1,074 \\
\hline $\mathrm{HF}$ & Randomly selected animals & 282 & - & - & 282 \\
\hline BV & Randomly selected animals & 280 & - & - & 280 \\
\hline
\end{tabular}

Genotypes for the nonsense-mutation (rs378824791) were obtained for 3,650 animals representing three different breeds (Fleckvieh (FV), Holstein-Friesian (HF) and Braunvieh (BV)) using a TaqMan ${ }^{\circledR}$ genotyping assay. The nonsense-mutation is homozygous in the affected calves only. The mutation does not segregate in Holstein-Friesian and Braunvieh. Note that animals carrying the disease haplotype were specifically added to Fleckvieh panel.

a different aetiological basis of the syndrome observed in the Fleckvieh calves.

Genome-wide association analysis followed by autozygosity mapping identified a common 1,023 kb segment of extended homozygosity on BTA 21 in the affected calves. Variants in two genes (CRIP1 and CRIP2) with functional similarity to $S L C 39 A 4[20,21]$ were excluded to be associated with the phenotype of the affected calves. Finally, genome-wide re-sequencing of one of the affected calves revealed a putatively causal loss-of-function mutation in the phospholipase D family member 4 encoding gene PLD4. Given the relatively low fold sequence coverage, some variants might have been wrongly called and lost, respectively. However, multi-sample variant calling followed by population-based genotype imputation as applied in

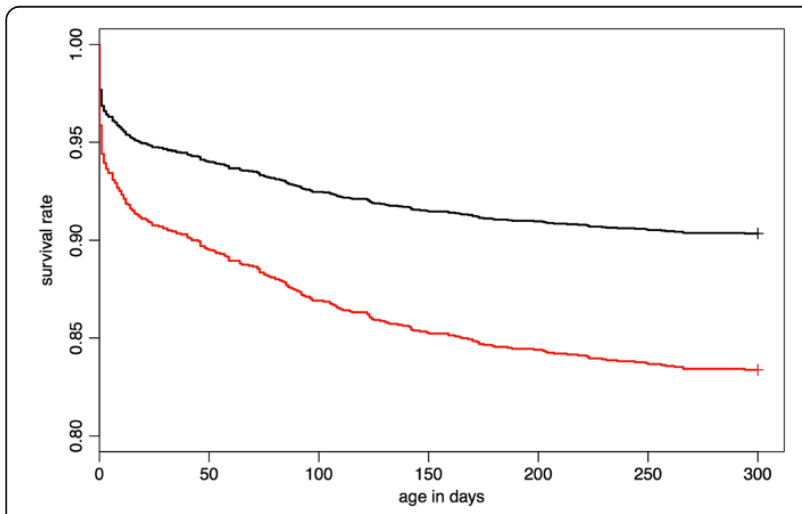

Figure 5 Survival analysis of calves from different mating types. Survival rate of calves from two different mating types (non-carrier [sire] $\times$ carrier [maternal grandsire], carrier [sire] $\times$ carrier [maternal grandsire]) as a function of the animals' age. The black line represents the survival rate of calves descending from matings where the maternal grandsire is a carrier while the sire does not carry the $p$. W215X-mutation. The red line represents the survival rate of calves descending from matings where both, sire and maternal grandsire are carriers of the mutation. the present study provides high quality genotypes even for lowly covered sites [14]. The p.W125X-mutation was perfectly associated in a panel of 3,650 animals, further substantiating our suspicion of causality.

Phospholipase D4 is a member of the family of phospholipid signalling enzymes [17] and is mainly expressed in spleen and early microglia, suggesting a role in immunological pathways [22,23]. Although PLD4 seems to be involved in phagocytosis, little is known about its enzymatic function in cells [22]. Recent studies revealed an association between variants in PLD4 and two autoimmune diseases in human, namely systemic sclerosis [23] and rheumatoid arthritis [24], disorders with inflammatory skin lesions [25] and interstitial lung diseases $[26,27]$. Furthermore, knockout mice with a nonsense mutation in PLD4 manifest a phenotype with thin hair and growth retardation [28]. In addition, Arun et al. [29] demonstrated the importance of phospholipase family D members in mediating the repair of plasma membrane disruptions in mice keratinocytes emphasising the role of these phospholipid signalling enzymes in membrane function and wound healing. A mutation in a phospholipase-domain containing protein (PNPLA1) results in a severe cornification disorder in dogs, providing evidence for a key role of lipases in the keratinisation process and the metabolism of the epidermal barrier in general [30]. Taken together, it seems very likely that an impaired function of PLD4 is causal for the severe skin lesions and the poor general health status of the affected calves.

Compared to the wild-type protein, the PLD4 protein is predicted to be shortened by 273 amino acids in the affected calves. The truncated protein may be retained with an impaired function and/or the transcript may be degraded via nonsense-mediated mRNA decay [31]. If the truncated protein is retained, its function is likely to be severely compromised as it lacks essential domains for enzymatic activity $[16,17]$. However, the actual effect of the 
Table 3 Survival rate of calves descending from different mating types

\begin{tabular}{|c|c|c|c|c|c|c|c|c|c|}
\hline \multicolumn{2}{|c|}{ Carrier-state for the p.W215X-mutation } & \multirow{2}{*}{$\begin{array}{l}\text { Number } \\
\text { of calves }\end{array}$} & \multicolumn{7}{|c|}{ Survival rate at different days } \\
\hline Sire & Maternal grandsire & & 6 & 10 & 20 & 50 & 102 & 202 & 300 \\
\hline Non-carrier & Carrier & 2,552 & 0.961 & 0.957 & 0.949 & 0.940 & 0.924 & 0.909 & 0.903 \\
\hline Carrier & Carrier & 1,213 & 0.931 & 0.923 & 0.911 & 0.895 & 0.869 & 0.843 & 0.834 \\
\hline
\end{tabular}

The survival rate of calves from different mating types was estimated using a Kaplan-Meier estimator.

mutation on the expression of PLD4 needs to be unravelled in subsequent studies.

Although there is a striking similarity in the phenotypic appearance of the Fleckvieh calves and Holstein-Friesian animals suffering from BHZD [4], there are no clues for a connection between PLD4 and zinc metabolism. Furthermore, zinc supplementation did not ameliorate any of the symptoms of the affected calves, corroborating that impaired zinc metabolism might not be causal for the phenotype. Based on our findings the tentative diagnosis of zinc deficiency can no longer be maintained.

Haplotype analysis revealed that three unaffected animals are homozygous for the associated haplotype. However, none of them was homozygous for the p. W215X-mutation. It seems likely that the mutation might have occurred in the germline of a recent founder animal, resulting in two identical haplotypes differing for the deleterious allele only. A similar situation has been observed for the arachnomelia syndrome (OMIA 0000599913) in Brown Swiss cattle [32]. Using array-derived genotypes does not allow distinguishing between animals carrying the affected and unaffected haplotype version. Thus, the haplotype frequency derived from array-based genotypes $(1.86 \%)$ is higher than the frequency of the p.W125X-mutation. The frequency of the p.W215Xmutation was estimated to $1.1 \%$ only in the current Fleckvieh population. However, the widespread use of a single bull carrying the defect allele could lead to a rapid increase of the frequency within few generations. Since array-based genotypes are routinely obtained for all candidate bulls, animals carrying the disease-associated haplotype can now be easily identified. Such animals should be directly genotyped for the p.W215X-mutation and animals carrying the mutation should be excluded from artificial insemination. This cost-effective approach will prevent unneeded animal suffering and economic losses by avoiding inadvertent carrier $\mathrm{x}$ carrier matings.

Assuming a frequency of $1.1 \%$ of the deleterious allele in the Fleckvieh population, equal use of all bulls and $\sim 1,000,000$ annual births, one would expect 121 affected calves per year. The actual number of expected cases is probably somewhat lower, as most carriers are related and farmers avoid close inbreeding. Furthermore, most of these cases will probably not be reported since the affected calves die due to rather unspecific diseases (e.g., enteritis or diarrhoea) before the manifestation of characteristic symptoms (i.e., hyperkeratotic and fissured skin). The significantly reduced survival rate of descendants from risk matings supports this assumption. However, incomplete penetrance could also reduce the observed incidences. Therefore it will be important to study animals being homozygous for the identified mutation from birth in a controlled environment for a better characterisation of the disorder.

\section{Conclusion}

A recessively inherited condition in Fleckvieh cattle resembles the phenotypic appearance of bovine hereditary zinc deficiency. Our results strongly support that a nonsense mutation (p.W215X) in the PLD4 encoding gene is causative for this disease in the Fleckvieh breed and that zinc deficiency is not involved in the aetiology of the disease. Further studies are necessary to unravel the detailed genotype-phenotype relationship. However, the identification of the causal variant allows for broad testing in the Fleckvieh population. Hence, an efficient management of this new defect is now possible.

\section{Methods}

\section{Animal ethics statement}

Semen samples were collected by approved commercial artificial insemination stations as part of their regular breeding and reproduction measures in cattle industry. The collection of blood samples was carried out by trained veterinarians during treatment of affected animals following standard veterinary protocols in Germany. No ethical approval was required for this study.

\section{Animals and DNA extraction}

In order to confirm a genetic predisposition to the observed phenotype, a case-control panel consisting of the eight affected calves, their dams and sires and twelve unaffected control animals was set up. Seven affected animals were identified and examined by veterinarians of the Clinic for Ruminants. One affected calf was reported by a herd veterinarian. Blood samples of the affected calves and their dams were collected by trained veterinarians following standard procedures and DNA was extracted using proteinase $\mathrm{K}$ digestion and salt-out extraction. For the control group, genomic DNA was prepared 
from semen straws following standard protocols using proteinase $\mathrm{K}$ digestion and phenol-chloroform extraction.

\section{Annotation and polymorphism screening of SLC39A4, CRIP1 and CRIP2}

The GENOMETHREADER software tool [33] was used to predict the genomic structure and localization of SLC39A4, CRIP1 and CRIP2 based on the University of Maryland UMD3.1 assembly of the bovine genome sequence [10] and the Dana-Farber Cancer Institute bovine gene index release 12.0 [34] together with the annotated RNA sequences of the UMD3.1 assembly [10]. The GENOMETHREADER output was viewed and edited using the Apollo sequence annotation editor [35]. The genes were PCR amplified (the primers are listed in Additional file 5), including exons, introns and the flanking 3'- and 5'-regions. Sequencing reactions were done using BigDye ${ }^{\bullet}$ Terminator v1.1 Cycle Sequencing Kit (Applied Biosystems, Foster, CA., USA; Life Technologies Corporation, USA). Electrophoresis of purified sequencing reactions was performed on the ABI 3130x1 Genetic Analyzer (Applied Biosystems, Foster, CA., USA; Life Technologies Corporation, USA). The Phred/Phrap/ Polyphred software suite [36-38] was used for base calling, sequence alignment and polymorphism detection. Sequences were viewed with consed [39].

\section{High- density genotyping and quality control}

The eight affected calves and 1,339 unaffected Fleckvieh bulls were genotyped with the Illumina BovineHD BeadChip comprising 777,962 SNPs. Genotype calling was performed using default parameters of Illumina's BeadStudio. The chromosomal position of the SNPs was determined based on the UMD3.1 assembly of the bovine genome [40]. 343 mitochondrial, 1,224 Y-chromosomal and 1,735 SNPs with unknown chromosome position were not considered for subsequent analyses. Quality control was carried out with PLINK v1.07 [41]. 19,734 SNPs for which genotyping failed in more than $5 \%$ of the individuals and 110,746 SNPs with minor allele frequency $<0.5 \%$ were omitted. Sporadically missing genotypes were imputed using Beagle genetic analysis software [42]. The final dataset comprised 1,347 animals (eight affected calves and 1,339 controls) and 644,450 SNPs.

\section{Genome-wide association study}

To account for population stratification and the resulting inflation of false positive associations, a mixed model based association analysis was performed. We used GEMMA [43] to fit the linear mixed model $\mathrm{y}=\mu+\mathrm{xb}+$ $\mathrm{Zu}+\mathrm{e}$, where y denotes the affection status (coded as 1 and 2 for affected and unaffected animals, respectively), $\mu$ is the intercept, $x$ is a vector of marker genotypes, $b$ is the SNP effect, $\mathrm{Z}$ is an incidence matrix, $\mathrm{u}$ is a vector of random polygenic effects $\sim \mathrm{N}\left(0, \mathrm{G} \sigma_{a}^{2}\right)$, where $\sigma_{a}^{2}$ is the additive genetic variance and $\mathrm{G}$ is the genomic relationship matrix (GRM) among the 1,347 animals built based upon 627,627 autosomal SNPs following VanRaden's approach [44] and e is a vector of error terms.

\section{Haplotype analysis}

Haplotypes of 1,347 animals of the initial genome-wide scan were inferred using default parameters of Beagle genetic analysis software (see above). To assess the frequency of the associated haplotype in a larger sample of the Fleckvieh population, array-derived genotypes of another 9,016 unaffected animals were analysed. Of the 9,016 animals, 7,000 were genotyped with the BovineSNP50 BeadChip (50 K) and 2,016 were genotyped with the Illumina BovineHD BeadChip (777 K). High-density genotypes and haplotypes of the $50 \mathrm{~K}$ data set were inferred based on haplotypes of the $777 \mathrm{~K}$ data set using a combination of Beagle (see above) and Minimac [45], which yields high imputation accuracy in cattle [46].

\section{Exploiting whole-genome re-sequencing data for mutation screening}

The genomes of 43 animals of the Fleckvieh population were sequenced to an average coverage of $7.46 \mathrm{x}$, among them an affected calf and a healthy animal being homozygous for the disease-associated haplotype with coverages of 7.8x and 6.2x, respectively. Sequencing on Illumina GA IIX and Hiseq 2000 instruments was performed as detailed by Jansen et al. [14]. Paired-end reads were obtained and mapped to the bovine reference sequence (see above) using the Burrows-Wheeler Aligner (BWA) [47]. PICARD (http://picard.sourceforge.net) was used to mark PCRduplicates. Subsequent multi-sample variant calling with mpileup [48] yielded genotypes at 17.17 million sites. Beagle phasing and imputation was applied to improve the primary genotype calls. A detailed overview of the entire variant calling pipeline and all obtained variants is presented in Jansen et al. [14]. Of 17.17 million sites, 7,086 SNPs and 574 InDels were located within the $1,023 \mathrm{~kb}$ segment $(70,550,045 \mathrm{bp}-71,573,501 \mathrm{bp})$ of extended homozygosity on BTA 21 . To account for inaccurately genotyped variants due to the low-coverage sequence data (e.g., mis-calling of heterozygous genotypes for rare variants $[49,50])$, we filtered for variants that were segregating (heterozygous or homozygous for the non-reference allele) in the affected animal, heterozygous or homozygous for the reference allele in the healthy animal being homozygous for the diseaseassociated haplotype and homozygous for the reference allele in 41 healthy animals. 


\section{5'-exonuclease diagnostic assay of the c.G645A-PLD4 mutation}

A 5'-exonuclease assay was developed to obtain genotypes for the nonsense mutation (c.G645A, p.W215X, Chr21:71,001,232 bp, rs378824791), using 5'-GGG CGG CAC ATC TAC GT-3' and 5'-CCA GGG CGG ACG AAC TC-3' as PCR primers, and 5'-CAT GGA CTG GCG GTC C-3' (wild type G allele) and 5'-CAT GGA CTG ACG GTC C-3' (mutant A allele) as probes (TaqMan ${ }^{\bullet}$, Life Technologies Corporation, USA). Reactions were carried out on an ABI7500 Real-Time PCR system (Life Technologies Corporation, USA) using standard procedures and analysed using the allelic discrimination endpoint analysis mode of the 7500 software package v2.0.5. Genotypes for the polymorphism were obtained in 3,650 animals representing three different breeds (Fleckvieh, Holstein-Friesian, Braunvieh).

\section{Survival analysis}

The survival rate of calves from two different mating types was estimated using a Kaplan-Meier estimator as implemented in the $\mathrm{R}$ package ('survival') [51]. For the control group the survival rate of 2,552 calves descending from matings where the sire does not carry the p.W215Xmutation whereas the maternal grandsire is a carrier S5 (non-risk matings) was estimated. The case group comprised 1,213 calves descending from matings where both sire and maternal grandsire carry the p.W215X-mutation (risk-matings).

\section{Availability of supporting data}

All relevant SNPs (with accession numbers of dbSNP) supporting the results of this article are included within the article and its additional files.

\section{Additional files}

Additional file 1: Overview of the identified SNPs in SLC39A4, CRIP1 and CRIP2.

Additional file 2: CNV-analysis within the segment of extended homozygosity. Signal intensities obtained from genotyping with the Illumina BovineHD BeadChip are displayed as log $\mathrm{R}$ ratios for cases and (randomly selected) controls within the segment of extended homozygosity. The log R ratio is displayed for 3-SNP-sliding windows.

Additional file 3: Genomic structure of the re-annotated PLD4 and INF2 genes. The genomic structure was predicted based on the University of Maryland UMD3.1 assembly of the bovine genome sequences [10] and the Dana-Farber Cancer Institute bovine gene index release 12.0 [33] by using GENOMETHREADER software tool. The GENOMETHREADER output was viewed and edited using Apollo sequence annotation editor [35].

Additional file 4: Visualisation of the p.W215X mutation in two apparently homozygous animals using the integrative genomics viewer (IGV, http://www.broadinstitute.org/igv/). Analysis of whole-genome re-sequencing data revealed a nonsense mutation in PLD4 as most likely causal for the disease of the affected calves. Re-sequencing confirmed that the mutation is homozygous in the affected calf.

Additional file 5: List of primer pairs for SLC39A4, CRIP1 and CRIP2.
Competing interests

The authors declare that they have no competing interests.

\section{Authors' contributions}

SJ, HP, NSG and RF conceived the study and participated in its design and coordination. SJ performed the sample preparation and carried out the molecular genetic experiments. HP and HS performed the statistical analyses. NSG clinically examined the affected calves and $M C L$ and $M M$ performed the pathological analyses. SJ, HP, NSG, MCL and RF drafted the manuscript. All authors read and approved the final manuscript.

\section{Acknowledgements}

We thank the Arbeitsgemeinschaft Süddeutscher Rinderzüchter und Besamungsorganisation e.V (ASR) and the Förderverein

Biotechnologieforschung e.V. (FBF) for sharing genotype data and the Bavarian State Research Center for Agriculture for providing additional genotypes and DNA samples. The study was supported by the German Federal Ministry of Education and Research (BMBF) within the AgroClustEr "Synbreed - Synergistic plant and animal breeding" (FKZ: 0315528A). We further acknowledge financial support from the Förderverein

Biotechnologieforschung e.V. (FBF) and the Universität Bayern e.V.

\section{Author details}

${ }^{1}$ Chair of Animal Breeding, Technische Universitaet Muenchen, 85354 Freising, Germany. ${ }^{2}$ Clinic for Ruminants with Ambulatory and Herd Health Services at the Centre for Clinical Veterinary Medicine,

Ludwig-Maximilians-Universitaet Muenchen, 85764 Oberschleissheim, Germany. ${ }^{3}$ Institute of Veterinary Pathology at the Centre for Clinical Veterinary Medicine, Ludwig- Maximilians-Universitaet Muenchen, 80539 Munich, Germany. ${ }^{4}$ ZuchtData EDV-Dienstleistungen GmbH, 1200 Wien, Austria.

Received: 28 February 2014 Accepted: 28 May 2014

Published: 22 July 2014

\section{References}

1. Online Mendelian Inheritance in Animals, OMIA, Faculty of Veterinary Science, University of Sydney (January 2014). http://omia.angis.org.au/.

2. Van Vleck LD: Effect of artificial insemination on frequency of undesirable recessive genes. J Dairy Sci 1967, 50:201-204

3. Charlier C, Coppieters W, Rollin F, Desmecht D, Agerholm JS, Cambisano N, Carta E, Dardano S, Dive M, Fasquelle C, Frennet J-C, Hanset R, Hubin X, Jorgensen C, Karim L, Kent M, Harvey K, Pearce BR, Simon P, Tama N, Nie H, Vandeputte S, Lien S, Longeri M, Fredholm M, Harvey RJ, Georges M: Highly effective SNP-based association mapping and management of recessive defects in livestock. Nat Genet 2008, 40:449-454.

4. Machen M, Montgomery T, Holland R, Braselton E, Dunstan R, Brewer G, Yuzbasiyan-Gurkan V: Bovine hereditary zinc deficiency: lethal trait A 46. J Vet Diagn Invest 1996, 8:219-227.

5. Brummerstedt E: Animal model of human disease: acrodermatitis enteropathica, zinc malabsorption. Am J Pathol 1977, 87:725-728.

6. Stöber M: Parakeratosis in black pied calves: 1: clinical findings and etiology. DTW Dtsch Tierarztl Wochenschr 1971, 78:257-265.

7. Wang K, Zhou B, Kuo Y-M, Zemansky J, Gitschier J: A novel member of a zinc transporter family is defective in acrodermatitis enteropathica. Am J Hum Genet 2002, 71:66-73.

8. Yuzbasiyan-Gurkan $V$, Bartlett E: Identification of a unique splice site variant in SLC39A4 in bovine hereditary zinc deficiency, lethal trait A46: an animal model of acrodermatitis enteropathica. Genomics 2006, 88:521-526.

9. Dufner-Beattie J, Weaver BP, Geiser J, Bilgen M, Larson M, Xu W, Andrews GK: The mouse acrodermatitis enteropathica gene SIc39a4 (Zip4) is essential for early development and heterozygosity causes hypersensitivity to zinc deficiency. Hum Mol Genet 2007, 16:1391-1399.

10. Florea L, Souvorov A, Kalbfleisch TS, Salzberg SL: Genome assembly has a major impact on gene content: a comparison of annotation in two bos taurus assemblies. PLoS One 2011, 6:e21400.

11. Hallquist NA, Khoo C, Cousins RJ: Lipopolysaccharide regulates cysteinerich intestinal protein, a zinc-finger protein, in immune cells and plasma. J Leukoc Biol 1996, 59:172-177.

12. Hempe JM, Cousins RJ: Cysteine-rich intestinal protein binds zinc during transmucosal zinc transport. Proc Natl Acad Sci USA 1991, 88:9671-9674. 
13. Cousins RJ, Lanningham-Foster L: Regulation of cysteine-rich intestinal protein, a zinc finger protein, by mediators of the immune response. J Infect Dis 2000, 182(Suppl 1):S81-84

14. Jansen $\mathrm{S}$, Aigner $\mathrm{B}$, Pausch $\mathrm{H}$, Wysocki $\mathrm{M}$, Eck $\mathrm{S}$, Benet-Pagès $\mathrm{A}$, Graf $\mathrm{E}$ Wieland T, Strom TM, Meitinger T, Fries R: Assessment of the genomic variation in a cattle population by re-sequencing of key animals at low to medium coverage. BMC Genomics 2013, 14:446.

15. Daetwyler HD, Capitan A, Pausch H, Stothard P, van Binsbergen R, Brøndum RF, Liao X, Djari A, Rodriguez SC, Grohs C, Esquerré D, Bouchez O, Rossignol M-N, Klopp C, Rocha D, Fritz S, Eggen A, Bowman PJ, Coote D, Chamberlain AJ, Anderson C, VanTassell CP, Hulsegge I, Goddard ME, Guldbrandtsen B, Lund MS, Veerkamp RF, Boichard DA, Fries R, Hayes BJ: Whole-genome sequencing of 234 bulls facilitates mapping of monogenic and complex traits in cattle. Nat Genet 2014. advance online publication: doi:10.1038/ng.3034

16. Xie Z, Ho WT, Exton JH: Association of the $\mathrm{N}$ - and C-terminal domains of phospholipase D: contribution of the conserved HKD motifs to the interaction and the requirement of the association for Ser/Thr phosphorylation of the enzyme. J Biol Chem 2000, 275:24962-24969.

17. Yoshikawa F, Banno Y, Otani Y, Yamaguchi Y, Nagakura-Takagi Y, Morita N, Sato Y, Saruta C, Nishibe H, Sadakata T, Shinoda Y, Hayashi K, Mishima Y, Baba H, Furuichi T: Phospholipase D family member 4, a transmembrane glycoprotein with no phospholipase D activity, expression in spleen and early postnatal microglia. PLoS One 2010, 5:e13932.

18. Trautwein G: Parakeratosis in black pied calves: 2: pathological findings. DTW Dtsch Tierarztl Wochenschr 1971, 78:265-270.

19. Maverakis E, Lynch PJ, Fazel N: Acrodermatitis enteropathica. Dermatol Online J 2007, 13:11.

20. O'Dell BL: Cysteine-rich intestinal protein (CRIP): a new intestinal zinc transport protein. Nutr Rev 1992, 50:232-233.

21. Cousins RJ: Gastrointestinal factors influencing zinc absorption and homeostasis. Int J Vitam Nutr Res 2010, 80:243-248.

22. Otani Y, Yamaguchi $Y$, Sato Y, Furuichi T, Ikenaka K, Kitani H, Baba H: PLD4 is involved in phagocytosis of microglia: expression and localization changes of PLD4 are correlated with activation state of microglia. PLOS One 2011, 6:e27544.

23. Terao C, Ohmura K, Kawaguchi Y, Nishimoto T, Kawasaki A, Takehara K, Furukawa H, Kochi Y, Ota Y, Ikari K, Sato S, Tohma S, Yamada R, Yamamoto K, Kubo M, Yamanaka H, Kuwana M, Tsuchiya N, Matsuda F, Mimori T: PLD4 as a novel susceptibility gene for systemic sclerosis in a Japanese population. Arthritis \& Rheumatism 2013, 65:472-480.

24. Okada Y, Terao C, Ikari K, Kochi Y, Ohmura K, Suzuki A, Kawaguchi T, Stahl EA, Kurreeman FAS, Nishida N, Ohmiya H, Myouzen K, Takahashi M, Sawada T, Nishioka Y, Yukioka M, Matsubara T, Wakitani S, Teshima R, Tohma S, Takasugi K, Shimada K, Murasawa A, Honjo S, Matsuo K, Tanaka H, Tajima K, Suzuki T, Iwamoto T, Kawamura Y, et al: Meta-analysis identifies nine new loci associated with rheumatoid arthritis in the Japanese population. Nat Genet 2012, 44:511-516.

25. Krieg T, Takehara K: Skin disease: a cardinal feature of systemic sclerosis. Rheumatology (Oxford) 2009, 3:iii14-18.

26. Picchianti Diamanti A, Germano V, Bizzi E, Laganà B, Migliore A: Interstitial lung disease in rheumatoid arthritis in the era of biologics. Pulm Med 2011, 2011:931342.

27. Bussone $G$, Mouthon L: Interstitial lung disease in systemic sclerosis. Autoimmun Rev 2011, 10:248-255.

28. Harris B, Ward-Bailey PF, Bergstrom DE, Bronson RT, Donahue LR: Thin hair with small size (thss) is a new recessive hair mutation on Chromosom 12. Bar Harbor, Maine: Mouse Mutant Resource Web Site, The Jackson Laboratory. (http://mousemutant.jax.org/). [September 17, 2012].

29. Arun SN, Xie D, Howard AC, Zhong Q, Zhong X, McNeil PL, Bollag WB: Cell wounding activates phospholipase $D$ in primary mouse keratinocytes. J Lipid Res 2013, 54:581-591.

30. Grall A, Guaguère E, Planchais S, Grond S, Bourrat E, Hausser I, Hitte C, Le Gallo M, Derbois C, Kim G-J, Lagoutte L, Degorce-Rubiales F, Radner FPW, Thomas A, Küry S, Bensignor E, Fontaine J, Pin D, Zimmermann R, Zechner R, Lathrop M, Galibert F, André C, Fischer J: PNPLA1 mutations cause autosomal recessive congenital ichthyosis in golden retriever dogs and humans. Nat Genet 2012, 44:140-147.

31. Frischmeyer PA, Dietz HC: Nonsense-mediated mRNA decay in health and disease. Hum Mol Genet 1999, 8:1893-1900.

32. Drögemüller $C$, Tetens J, Sigurdsson $S$, Gentile A, Testoni $S$, Lindblad-Toh K, Leeb T: Identification of the Bovine Arachnomelia Mutation by Massively
Parallel Sequencing Implicates Sulfite Oxidase (SUOX) in bone development. PLoS Genet 2010, 6:e1001079.

33. Gremme G, Brendel V, Sparks ME, Kurtz S: Engineering a software tool for gene structure prediction in higher organisms. Inform Software Tech 2005, 47:965-978.

34. Quackenbush J, Cho J, Lee D, Liang F, Holt I, Karamycheva S, Parvizi B, Pertea G, Sultana R, White J: The TIGR Gene Indices: analysis of gene transcript sequences in highly sampled eukaryotic species. Nucl Acids Res 2001, 29:159-164

35. Lewis SE, Searle SMJ, Harris N, Gibson M, lyer V, Richter J, Wiel C, Bayraktaroglu L, Birney E, Crosby MA, Kaminker JS, Matthews BB, Prochnik SE, Smith CD, Tupy U, Rubin GM, Misra S, Mungall CJ, Clamp ME: Apollo: a sequence annotation editor. Genome Biol 2002, 3:research0082. 1 - 0082.14.

36. Ewing B, Green P: Base-calling of automated sequencer traces using phred: Il: error probabilities. Genome Res 1998, 8:186-194.

37. Ewing B, Hillier L, Wendl MC, Green P: Base-calling of automated sequencer traces using phred: I: accuracy assessment. Genome Res 1998, 8:175-185.

38. Nickerson DA, Tobe VO, Taylor SL: PolyPhred: automating the detection and genotyping of single nucleotide substitutions using fluorescencebased resequencing. Nucleic Acids Res 1997, 25:2745-2751.

39. Gordon D, Abajian C, Green P: Consed: a graphical tool for sequence finishing. Genome Res 1998, 8:195-202.

40. Zimin AV, Delcher AL, Florea L, Kelley DR, Schatz MC, Puiu D, Hanrahan F, Pertea G, Van Tassell CP, Sonstegard TS, Marçais G, Roberts M, Subramanian $P$, Yorke JA, Salzberg SL: A whole-genome assembly of the domestic cow: bos taurus. Genome Biol 2009, 10:R42

41. Purcell $S$, Neale B, Todd-Brown $K$, Thomas $L$, Ferreira MAR, Bender D, Maller J, Sklar P, de Bakker PIW, Daly MJ, Sham PC: PLINK: a tool set for wholegenome association and population-based linkage analyses. Am J Hum Genet 2007, 81:559-575.

42. Browning $B L$, Browning SR: A unified approach to genotype imputation and haplotype-phase inference for large data sets of trios and unrelated individuals. Am J Hum Genet 2009, 84:210-223.

43. Zhou X, Stephens M: Genome-wide efficient mixed-model analysis for association studies. Nat Genet 2012, 44:821-824.

44. VanRaden PM: Efficient methods to compute genomic predictions. J Dairy Sci 2008, 91:4414-4423.

45. Howie B, Fuchsberger C, Stephens M, Marchini J, Abecasis GR: Fast and accurate genotype imputation in genome-wide association studies through pre-phasing. Nat Genet 2012, 44:955-959.

46. Pausch H, Aigner B, Emmerling R, Edel C, Götz K-U, Fries R: Imputation of high-density genotypes in the Fleckvieh cattle population. Genet Sel Evol 2013, 45:3

47. Li H, Durbin R: Fast and accurate short read alignment with BurrowsWheeler transform. Bioinformatics 2009, 25:1754-1760.

48. Li H, Handsaker B, Wysoker A, Fennell T, Ruan J, Homer N, Marth G, Abecasis $G$, Durbin R: The sequence alignment/map format and SAMtools. Bioinformatics 2009, 25:2078-2079.

49. Li Y, Sidore C, Kang HM, Boehnke M, Abecasis GR: Low-coverage sequencing: implications for design of complex trait association studies. Genome Res 2011, 21:940-951.

50. DePristo MA, Banks E, Poplin R, Garimella KV, Maguire JR, Hartl C, Philippakis AA, del Angel G, Rivas MA, Hanna M, McKenna A, Fennell TJ, Kernytsky AM, Sivachenko AY, Cibulskis K, Gabriel SB, Altshuler D, Daly MJ: A framework for variation discovery and genotyping using next-generation DNA sequencing data. Nat Genet 2011, 43:491-498.

51. Therneau TM, Grambsch PM: Modeling Survival Data: Extending the Cox Model. New York: Springer; 2000.

doi:10.1186/1471-2164-15-623

Cite this article as: Jung et al:: A nonsense mutation in PLD4 is associated with a zinc deficiency-like syndrome in Fleckvieh cattle. BMC Genomics 2014 15:623. 